\title{
Prehospital notification can effectively reduce in-hospital delay for thrombolysis in acute stroke
}

\author{
Elyar Sadeghi-Hokmabadi*,1, Mehdi Farhoudi ${ }^{1}$, Aliakbar Taheraghdam¹, Reza Rikhtegar ${ }^{1}$, \\ Rouzbeh Rajaei Ghafouri ${ }^{2}$, Rogayyeh Asadi ${ }^{1}$, Elham Mehdizadeh Far ${ }^{1}$, Neda Ghaemian ${ }^{1}$, \\ Mehrdad Mehrara ${ }^{1}$ \& Reshad Mirnour ${ }^{1}$ \\ ${ }^{1}$ Neurosciences Research Center (NSRC), Neurology Department, Imam Reza Hospital, Tabriz University of Medical Sciences, Tabriz, \\ Iran \\ ${ }^{2}$ Emergency medicine research team, Emergency Department, Imam Reza Hospital, Tabriz University of Medical Sciences, Tabriz, \\ Iran \\ * Author for correspondence: Tel.: +98 914311 0367, Fax: +98 411334 0730; aeass@yahoo.com
}

\begin{abstract}
Aim: To reduce in-hospital intervals by developing a prehospital notification (PHN) protocol which directly notifies a neurologist to prepare for possible treatment. Methods: A 24/7 connection was established between emergency medical services dispatch and the on-call neurologist. A database of all patients with in-hospital stroke code activation was developed, door-to-computed tomography (CT) time and door-toneedle time was recorded from January 2013 to December 2016. The statistical results were considered significant at $p<0.05$. Result: PHN resulted in a significant reduction in door-to-CT time (median 14 vs 20; $p<0.001$ ). Among patients who were treated with intravenous thrombolysis, door-to-needle time was significantly shorter in patients with PHN compared with non-PHN group (median 42 vs $70 ; p<0.001$ ). Conclusion: PHN effectively reduced door-to-CT and door-to-needle times.
\end{abstract}

First draft submitted: 24 October 2017; Accepted for publication: 4 December 2017; Published online: 17 January 2018

Keywords: door-to-CT $\bullet$ door-to-needle $\bullet$ golden hour $\bullet$ in-hospital delay $\bullet$ prehospital notification $\bullet$ stroke $\bullet$ stroke code $\bullet$ stroke outcome $\bullet$ symptom onset $\bullet$ thrombolysis

Stroke is a major cause of mortality and morbidity [1]. Intravenous tissue plasminogen activator (IV-tPA) remains a major available treatment option for acute stroke in developing countries where other methods such as thrombectomy are limited [2]. However, it has been proven to reduce the combined end point of death and disability after stroke if given within the golden hour after the onset of symptoms [3,4]. Its benefit is strongly time dependent [5] and multiple studies have shown that earlier treatment is associated with better outcomes [6-8]. Consensus guidelines recommend a target time of $<25 \mathrm{~min}$ from hospital arrival to CT scan and $<60 \mathrm{~min}$ from hospital arrival to treatment [9]. Unfortunately, due to significant delay in assessment of stroke patients in developing countries, the golden hour is often missed and patients lose the opportunity to be treated promptly [2].

There are numerous contributing factors to delay treatment of stroke. These can be divided into 'out of hospital' and 'in-hospital' delays and since decades ago, lots of studies have been conducted to evaluate such delays and therefore improving the outcome of stroke. Review of these studies shows that over time there has been a steady reduction in such time intervals [10].

Although major delays happen in the recognition of symptoms and transferring the patients to hospitals, Inhospital delays are also important in overall treatment failure. Multiple factors have been identified for in-hospital delays including decision making process, laboratory tests, neurology service arrival and imaging [10-12]. Previously, we tried to identify and resolve the issues related to in-hospital latencies in our hospital in Tabriz, Iran. We successfully implemented simple and effective methods to enhance in-hospital efficacy for timely management of acute stroke [2]. 
One simple and cost-effective measure to tackle in-hospital delay is prehospital notification (PHN) by the emergency medical services (EMS), which allows faster activation of the stroke team and has shown to be effective in reducing in-hospital delays [13-15].

Prenotification by contacting a neurologist directly, has been less studied $[13,14,16]$. The aim of this study was to compare in-hospital time intervals in stroke patients treated with IV-tPA with and without PHN.

\section{Methods}

\section{Setting}

Before commencing the study, the EMS personnel were trained for PHN of stroke patients. This study was retrospectively conducted with prospectively collected data from January 2013 (after establishment of the protocol) to December 2016 at Imam Reza Hospital in the city of Tabriz, Iran. During this period, all patients for whom the stroke code was activated were included in the study and patients were divided into two groups, with PHN and without PHN.

This hospital is a tertiary university center with an annual stroke admission of 900 patients, located in Tabriz in the province of East Azerbaijan, a city with an area of $45,700 \mathrm{~km}^{2}$ and a population of 3.7 million (2011 census). The hospital is the only center in the province which provides $24 / 7$ neurological services for thrombolysis. Endovascular treatment for acute stroke is not available in this center and none of the patients underwent thrombectomy or intra-arterial thrombolysis.

\section{Protocol for PHN}

The PHN protocol was established in December 2012. For this protocol, EMS personnel were initially trained. Once EMS personnel visit a patient with signs of probable stroke according to the Cincinnati stroke scale, where the onset of symptoms is less than $3 \mathrm{~h}$, they notify EMS dispatch. The dispatch then directly contacts the on-call stroke neurologist, who is available $24 / 7$ by mobile phone. The neurologist activates the hospital's stroke code by contacting the neurology resident and emergency physician at the hospital.

For all the patients, the stroke code was activated either before hospital arrival (PHN) or upon/after emergency department (ED) arrival (no-PHN). The patients in the 'no-PHN' group were those who were mainly transferred to hospital by family members or relatives. The in-hospital process after stroke code activation has been detailed elsewhere [2]. In all the cases, the imaging used for evaluation was a CT scan.

\section{EMS training}

All EMS and dispatch personnel were divided into three groups based on work shifts. Each group attended a 1-h training session lectured by a stroke neurologist. The importance of timing for early treatment of stroke patients was strongly emphasized to create a sense of urgency for treatment of these patients. The signs and symptoms of stroke according to the Cincinnati stroke scale were discussed. These training courses were repeated every 6 months.

\section{Study purpose}

The primary purpose of the study was to: determine the difference of door-to-CT time for patients with and without PHN; and to determine the difference of door-to-needle time for patients with and without PHN (in case of IV thrombolysis).

\section{Study variables}

The data were recorded for all patients prospectively for demographics (age and gender), interval of symptom onset to hospital arrival time and door-to-CT time. For patients who were treated with a thrombolytic agent, door-toneedle time and baseline stroke severity as assessed by the National Institutes of Health Stroke Scale (NIHSS) was also recorded.

\section{Statistical analysis}

Statistical analysis was performed using SPSS version 21 (for Windows). Chi-square or the Fisher Exact tests were used for categorical variables. Because of the non-normal distribution of continuous variables, the Mann-Whitney $\mathrm{U}$ test was carried out. The statistical test results were considered significant at $\mathrm{p}<0.05$. The data were presented as median and interquartile range. 


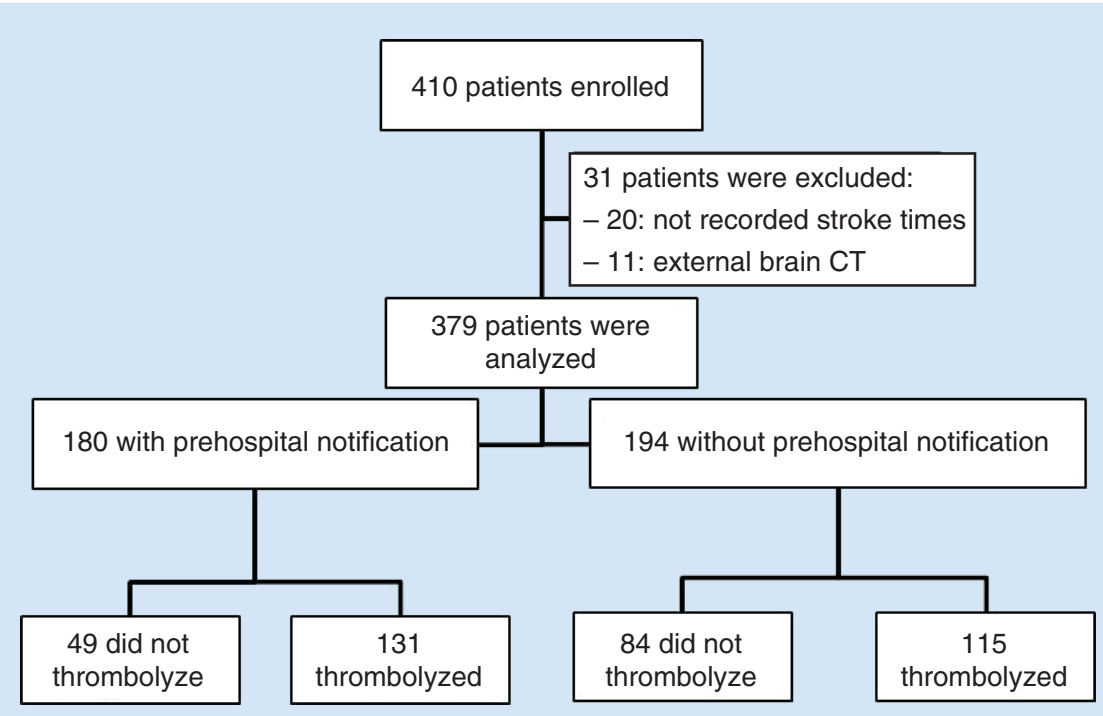

Figure 1. Distribution of participants.

Table 1. Baseline characteristics of intravenous r-tissue plasminogen activator treated patients regarding prehospital notification status.

\begin{tabular}{|c|c|c|c|}
\hline \multirow[t]{2}{*}{ Characteristics } & \multicolumn{2}{|c|}{ Prehospital notification } & \multirow[t]{2}{*}{ p-value } \\
\hline & Yes (n D 131) & No (n D 115) & \\
\hline Age, years and median (IQR) & $67(58-75)$ & $66(55-77)$ & 0.274 \\
\hline Male, n (\%) & $66(57.4 \%)$ & $72(55.0 \%)$ & 0.702 \\
\hline Baseline National Institutes of Health Stroke Scale, median (IQR) & $15(10-20)$ & $11(7.5-17)$ & $<0.001$ \\
\hline Onset to emergency department arrival time (min) & $83(55-121)$ & $78(50-120)$ & 0.462 \\
\hline Door-to-CT time, min and median (IQR) & $15(10-25)$ & $25(18-45)$ & $<0.001$ \\
\hline Door-to-needle time, min, median (IQR) & $42(34-56)$ & $70(53-95)$ & $<0.001$ \\
\hline
\end{tabular}

\section{Ethics}

This study was approved by the Regional Ethics Committee of Tabriz University of Medical Sciences. Informed consent was obtained from participants or their next of kin.

\section{Results}

During the study period, the ED was notified of a total of 410 patients for which the stroke code was activated (with or without PHN). Three patients were transferred to the hospital by helicopter EMS. Of the 410 patients, 31 were excluded. The stroke timings were not recorded for 20 patients and 11 patients had brain imaging performed elsewhere before hospital arrival. The analysis was carried out for the remaining 379 patients, 180 with PHN and 199 without PHN (Figure 1).

Median door-to-CT time for the PHN group was 6 min less than for the no-PHN group, which was statistically significant (median [interquartile range: IQR]; 14 [9-22] vs 20 [14-35]; $\mathrm{p}<0.001$ ).

Overall, $64.9 \%(246 / 379)$ of all patients were treated with IV thrombolysis (IVT). Table 1 shows baseline characteristics of patients treated with IV recombinant tissue plasminogen activator (r-TPA) associated with PHN. Among patients treated with IVT, the median door-to-CT and door-to-needle time was significantly lower for the PHN group compared with those in the no-PHN group (median [IQR]; 15 [10-22] vs 25 [18-45]; p < 0.001 and median [IQR]; 42 [34-56] vs 70 [53-95]; p < 0.001, respectively). Of all treated with thrombolysis, 17 (13\%) in $\mathrm{PHN}$ and 35 (30\%) in non-PHN had a symptom onset of more than $3 \mathrm{~h}$.

This study also evaluated the accuracy of code activation by EMS technicians. The EMS technicians were instructed based on the protocol to notify dispatch if they were visiting a patient who met the Cincinnati stroke 


\begin{tabular}{|c|c|c|c|}
\hline \multirow[t]{2}{*}{ Contraindication } & \multicolumn{2}{|c|}{ Prehospital notification } & \multirow[t]{2}{*}{ Total } \\
\hline & No & Yes & \\
\hline Intracranial hemorrhage & 14 & 14 & 28 \\
\hline Minor stroke & 5 & 1 & 6 \\
\hline Transient ischemic attack or rapidly improving & 12 & 4 & 16 \\
\hline Large stroke (more than a third) & 2 & 1 & 3 \\
\hline Awakening stroke & 8 & 6 & 14 \\
\hline Patients refused treatment & 6 & 2 & 8 \\
\hline Nonstroke diagnosis & 6 & 7 & 6 \\
\hline International normalized ratio $>1.7$ & 2 & 0 & 2 \\
\hline Symptom onset $>$ out of treatment window & 11 & 6 & 17 \\
\hline Refractory hypertension & 1 & 3 & 4 \\
\hline Age $>80$ & 10 & 4 & 14 \\
\hline Medication not available & 1 & 0 & 1 \\
\hline Active gastrointestinal bleeding & 1 & 0 & 1 \\
\hline Recent myocardial infarction & 1 & 1 & 2 \\
\hline Recent stroke & 1 & 0 & 1 \\
\hline 3-4.5 $\mathrm{h}$ and National Institutes of Health Stroke Scale $>25$ & 1 & 0 & 1 \\
\hline Intraparenchymal mass & 2 & 0 & 2 \\
\hline Total & 84 & 49 & 133 \\
\hline
\end{tabular}

scale and the onset of symptoms or the time the patient was last known to be normal was less than $3 \mathrm{~h}$. The code activation was correct in $89.4 \%(161 / 180)$ of patients. Among the remaining $19(10.6 \%)$ patients, six had actual onset times more than what was recommended in guidelines, six had experienced stroke on awakening and the code was activated for seven patients based on items that were not part of the Cincinnati stroke scale (generalized weakness, loss of consciousness and dizziness, etc.). Table 2 shows the contraindications for administering IVT in both groups. Based on recent guidelines [9,17], IVT is not recommended in patients presenting in $3-4.5 \mathrm{~h}$ window and with a severe stroke (NIHSS >25). As a result, thrombolysis was not given to one patient who fell into this category in no-PMH group.

\section{Discussion}

This study demonstrates that educating EMS personnel and implementing PHN with a direct 24/7 telephone hotline between the EMS dispatch and a neurologist reduced door-to-CT and door-to-needle times for acute stroke patients. Previous studies have reported similar results about the effect of PHN on in-hospital time targets [18-25].

The ED of Imam Reza Hospital is very crowded because it is the only referral center in the province for acute vascular patients, trauma and other emergencies. The advantage of the protocol used in this study was the direct contact between EMS and the on-call neurologist who is responsible for notifying the CT room, lab center and stroke unit. This decreased the load on the ED physician, who is routinely overburdened with emergency referrals. Furthermore, since the attending neurologist is involved from the beginning, the stroke unit has more time to get prepared before patient arrival and even more time available to transfer a patient, if necessary.

Based on stroke registry (established in 2015) data in our hospital, annual number of stroke admission is about 900 patients. Nevertheless, more than 410 patients were treated with IVT during a 4-year period. The reason for the huge gap between the total admission and stroke code activation is the majority of admitted patients present after the 'golden hour' for thrombolysis due to out of hospital delays. It is notable that a recent study at the center found that only $31 \%$ of patients with acute stroke arrived at the ED within $3 \mathrm{~h}$ of symptom onset [26]. Although implementation of the stroke code system and PHN are important interventions to reduce onset to treatment time, the severe lack of public awareness to recognize stroke symptoms is the reason why thrombolysis rate does not rise effectively. In a recent report, the rate of thrombolysis for acute stroke patients was less than $5 \%$ at this center [27]; therefore, public education is crucial to reach a higher rate of thrombolysis. 
Response to IVT is time dependent and its effect on a good outcome is negatively influenced by treatment delay [28]. Based on the data from hospitals participating in the Get With the Guidelines-Stroke Program, among 25,504 patients treated with IVT, every 15 min reduction in door-to-needle time was associated with $5 \%$ lower odds of mortality [29]. So by implementing PHN system and reducing the door-to-needle time, the probability of a good outcome could be increased.

For hospitals with stroke centers that have not yet implemented the PHN system, we strongly suggest to do so, because its implementation is easy and cheap, it does not need any specific infrastructure and its effects reflect in the short term.

Overall, PHN by EMS was correct for $89 \%$ of patients, which indicated that the 1-h training sessions every 6 months for EMS personnel was efficient, but in 19 patients, the EMS incorrectly prenotified dispatch hence the trainers should emphasize on the importance of taking more detailed history for precise determination of symptoms onset.

It is very important to note that stroke severity plays an important role in 'prehospital delays' or 'symptom onset to seek medical help' times [30]. As mentioned before, the aim of this study was not to evaluate such delays, but to reduce the 'in-hospital time' intervals. Moreover, we did not consider stroke risk factors as these may have little impact on our variables.

In a study by Sauser $e$ t al., the door-to-imaging time was reduced $0.31 \mathrm{~min}$ per NIHSS score, but they could not demonstrate any significant relation between door-to-needle time and severity [31]. In a multicenter study involving 5563 patients, stroke severity was directly associated with thrombolysis delay. However, in this study, PHN was not considered and patients were included from a stroke registry regardless of their method of arrival and any PHN [32]. It seems that PMH can reduce in-hospital delay as the neurologist will arrange for prompt imaging, laboratory tests and an imminent thrombolysis therefore 'stroke severity' appears to have a limited role in such setting. However, we agree that stroke severity can affect intervals in 'no-PHN' group.

This study had some limitations. First, it was carried out at a single center with a limited number of cases; the results may not be applicable to other health systems. More broad multicenter research is necessary to validate the results and determine whether shortening of the door-to-needle time by PHN lead to better outcomes for the patients. Second, the staffs were aware of eligible patients who presented with stroke symptoms within the 3-h window but EMS personnel missed for notification, but specific data are not available for these patients. Third, the total number of stroke admission, the stroke code and temporal windows rate are not available for this study, but Tabriz stroke registry program is running since 1 January 2015 and we have more detailed data since then.

\section{Conclusion}

The results of this study show that simple, brief and continuing training sessions for EMS personnel and implementation of PHN by direct 24/7 telephone contact between dispatch and a neurologist effectively reduced door-to-CT and door-to-needle times for acute stroke patients.

\section{Summary points}

- The aim was to study the effect of prehospital notification (PHN) on in-hospital time interval and to improve the stroke management outcome in developing countries.

- The emergency medical services (EMS) personnel were trained before starting the study.

- A 24/7 phone line connection was established between EMS and the on-call neurologist.

- All data recorded between 2013 and 2016 were reviewed.

- The analysis was carried out for 379 patients, 180 with PHN and 199 without PHN.

- PHN by EMS, resulted in a significant reduction in door-to-CT time.

- Door-to-needle time was significantly shorter in patients with PHN.

- The code activation by EMS was correct in $89.4 \%(161 / 180)$ of patients. 
No writing assistance was utilized in the production of this manuscript.

\section{Ethical conduct of research}

The authors state that they have obtained appropriate institutional review board approval or have followed the principles outlined in the Declaration of Helsinki for all human or animal experimental investigations. In addition, for investigations involving human subjects, informed consent has been obtained from the participants involved.

\section{Open access}

This work is licensed under the Attribution-NonCommercial-NoDerivatives 4.0 Unported License. To view a copy of this license, visit http://creativecommons.org/licenses/by-nc-nd/4.0/

\section{References}

1 Rothwell PM, Coull AJ, Silver LE et al. Population-based study of event-rate, incidence, case fatality and mortality for all acute vascular events in all arterial territories (Oxford Vascular Study). Lancet 366, 1773-1783 (2005).

2 Sadeghi-Hokmabadi E, Taheraghdam A, Hashemilar M et al. Simple in-hospital interventions to reduce door-to-CT time in acute stroke. Int. J. Vasc. Med. 2016, 1656212 (2016).

3 Lees K, Bluhmki E, von Kummer R et al. Time to treatment with intravenous alteplase and outcome in stroke: an updated pooled analysis of ECASS, ATLANTIS, NINDS and EPITHET. Lancet 375(9727), 1695-1703 (2010).

4 Pashapour A, Atalu A, Farhoudi M et al. Early and intermediate prognosis of intravenous thrombolytic therapy in acute ischemic stroke subtypes according to the causative classification of stroke system. Pak. J. Med. Sci. 29(1), 181-186 (2013).

5 Wardlaw JM, Murray V, Berge E et al. Recombinant tissue plasminogen activator for acute ischaemic stroke: an updated systematic review and meta-analysis. Lancet 379(9834), 2364-2372 (2012).

6 Strbian D, Soinne, Sairanen et al. Ultraearly thrombolysis in acute ischemic stroke is associated with better outcome and lower mortality. Stroke 41, 712-716 (2010).

7 Marler JR, Tilley BC, Lu M et al. Early stroke treatment associated with better outcome. The NINDS rt-PA Stroke Study. Neurology 55(11), 1649-1655 (2000).

8 Tsivgoulis G, Katsanos AH, Kadlecová P. Intravenous thrombolysis for ischemic stroke in the golden hour: propensity-matched analysis from the SITS-EAST registry. J. Neurol. 264(5), 912-920 (2017).

9 Jauch EC, Saver JL, Adams HP Jr et al. Guidelines for the early management of patients with acute ischemic stroke: a guideline for healthcare professionals from the American Heart Association/American Stroke Association. Stroke 44(3), 870-947 (2013).

10 Metts EL, Bailey AM, Weant KA, Justice SB. Identification of rate-limiting steps in the provision of thrombolytics for acute ischemic stroke. J. Pharm. Pract. 30(6), 606-611 (2017).

11 Huang Q, Ma QF, Feng J, Cheng WY, Jia JP, Song HQ. Factors associated with in-hospital delay in intravenous thrombolysis for acute ischemic stroke: lessons from China. PLoS ONE 10(11), e0143145 (2015).

12 Evenson KR, Foraker RE, Morris DL, Rosamond WD. A comprehensive review of prehospital and in-hospital delay times in acute stroke care. Int. J. Stroke 4(3), 187-199 (2009).

13 Binning MJ, Sanfillippo G, Rosen W et al. the neurological emergency room and prehospital stroke alert: the whole is greater than the sum of its parts. Neurosurgery 74(3), 281-285 (2014).

14 Casolla B, Bodenant M, Girot M, Cordonnier C, Pruvo JP, Wiel E. Intra-hospital delays in stroke patients treated with rt-PA: impact of preadmission notification. J. Neurol. 260(2), 635-639 (2013).

15 Kim SK, Lee SY, Bae HJ et al. Pre-hospital notification reduced the door-to-needle time for iv t-PA in acute ischaemic stroke. Eur. J. Neurol. 16(12), 1331-1335 (2009).

16 Belvís R, Cocho D, Martí-Fàbregas J et al. Benefits of a prehospital stroke code system. Feasibility and efficacy in the first year of clinical practice in Barcelona, Spain. Cerebrovasc. Dis. 19(2), 96-101 (2005).

17 Demaerschalk BM, Kleindorfer DO, Adeoye OM et al. Scientific rationale for the inclusion and exclusion criteria for intravenous alteplase in acute ischemic stroke: a statement for healthcare professionals from the American Heart Association/American Stroke Association. Stroke 47(2), 581-641 (2016).

18 Abdullah AR, Smith EE, Biddinger PD, Kalenderian D, Schwamm LH. Advance hospital notification by EMS in acute stroke is associated with shorter door-to-computed tomography time and increased likelihood of administration of tissue-plasminogen activator. Prehosp. Emerg. Care. 12(4), 426-431 (2008).

19 Quain DA, Parsons MW, Loudfoot AR et al. Improving access to acute stroke therapies: a controlled trial of organised prehospital and emergency care. Med. J. Aust. 189(8), 429-433 (2008). 
20 O'Brien W, Crimmins D, Donaldson W et al. FASTER (Face, Arm, Speech, Time and Emergency Response): experience of Central Coast Stroke Services implementation of a prehospital notification system for expedient management of acute stroke. J. Clin. Neurosci. 19(2), 241-245 (2012).

21 Meretoja A, Strbian D, Mustanoja S et al. Reducing in-hospital delay to 20 minutes in stroke thrombolysis. Neurology 79(4), 306-313 (2012).

22 Gladstone DJ, Rodan LH, Sahlas DJ et al. A citywide prehospital protocol increases access to stroke thrombolysis in Toronto. Stroke 40(12), 3841-3844 (2009).

23 Patel MD, Rose KM, O’Brien EC, Rosamond WD. Prehospital notification by emergency medical services reduces delays in stroke evaluation. Findings from the North Carolina stroke care collaborative. Stroke 42(8), 2263-2268 (2011).

24 Ragoschke-Schumm A, Walter S, Haass A et al. Translation of the 'time is brain' concept into clinical practice: focus on prehospital stroke management. Int. J. Stroke. 9(3), 333-340 (2014).

25 Baldereschi M, Piccardi B, Di Carlo A et al. Relevance of prehospital stroke code activation for acute treatment measures in stroke care: a review. Cerebrovasc. Dis. 34(3), 182-190 (2012).

26 Ayromlou $\mathrm{H}$, Soleimanpour $\mathrm{H}$, Farhoudi $\mathrm{M}$ et al. Eligibility assessment for intravenous thrombolytic therapy in acute ischemic stroke patients; evaluating barriers for implementation. Iran. Red. Crescent. Med. J. 16(5), e11284 (2014).

27 Sadeghi-Hokmabadi E, Farhoudi M, Taheraghdam A et al. Intravenous recombinant tissue plasminogen activator for acute ischemic stroke: a feasibility and safety study. Int. J. Gen Med. 9, 361-367 (2016).

28 Emberson J, Lees KR, Lyden P et al. Effect of treatment delay, age, and stroke severity on the effects of intravenous thrombolysis with alteplase for acute ischaemic stroke: a meta-analysis of individual patient data from randomized trials. Lancet 384(9958), 1929-1935 (2014).

29 Fonarow GC, Smith EE, Saver JL et al. Timeliness of tissue-type plasminogen activator therapy in acute ischemic stroke: patient characteristics, hospital factors and outcomes associated with door-to-needle times within 60 minutes. Circulation 123(7), 750-758 (2011).

30 Faiz KW, Sundseth A, Thommessen B et al. Factors related to decision delay in acute stroke. J. Stroke Cerebrovasc. Dis. 23(3), 534-539 (2014).

31 Sauser K, Levine DA, Nickles AV, Reeves MJ. Hospital variation in thrombolysis times among patients with acute ischemic stroke: the contributions of door-to-imaging time and imaging-to-needle time. JAMA Neurol. 71(9), 1155-1161 (2014).

32 Mikulík R, Kadlecová P, Czlonkowska A et al. Factors influencing in-hospital delay in treatment with intravenous thrombolysis. Stroke 43(6), 1578-1583 (2012). 
